\title{
CFD-simulation of gas-solid flow in bubbling fluidized bed reactor
}

\author{
Oumaima El ajouri ${ }^{1, *}$, Mohamed Lhassane Lahlaouti $^{1}$, and Bousselham Kharbouch $^{1}$ \\ ${ }^{1}$ Laboratory of energy and fluid mechanics, Faculty of sciences of Tetouan, Abdelmalek Essaadi University, Tetouan 93002, Morocco
}

\begin{abstract}
The hydrodynamic behaviour of gas-solid flow has been studied in a two-dimensional bubbling fluidized bed reactor filled with particles of $275 \mu \mathrm{m}$ in size, using computational fluid dynamics (CFD). The two-fluid model (TFM) approach based on the concept of Eulerian-Eulerian in combination with Kinetic Theory of Granular Flow (KTGF) is used to represent the fluid mechanics involved in the flow. The computational implementation was realized by the commercial software ANSYS FLUENT. Interphase momentum exchange between gas and solid phases were calculated using the modified Syamlal O'Brien drag force function. The simulation predictions have been comprehensively validated with experimental data measurements of bed expansion rate, pressure drops and average gas volume fraction profiles. The pressure drops predicted by the simulations were in comparatively strong agreement with the experimental data at higher superficial gas velocities. The bed expansion ratio of the simulation results is closer to that of the experimental data. This work provides a scalable means to assist in the design and operation of fluidised bed reactors and fluidisation processes.
\end{abstract}

\section{Introduction}

In recent years, due to its renewable, durable and environmentally friendly nature of biomass energy production has gained a great deal of interest within the research scientific community and the industrial engineering sector. Biomass fuel can reduce dependence on energy production via fossil fuels, thus reducing the associated adverse environmental impacts [1,2]. Biomass gasification is always faced with a variety of technical challenges, pushing back potential commercialization. Among these challenges are the propagation of the complex chemical reactions within the fluidization zone and the limitations imposed by the process control and the prediction difficulty of momentum models due to the turbulent flow behaviour of the gas and the flowing particles [3].

The complicated processes - inherently associated with integrated limits - would almost economically and ecologically render it unviable to study all the factors involved experimentally and, therefore, would require the development of reliable mathematical models [4]. The fluidized bed reactors can facilitate the study of thermochemical processes associated with biomass gasification, also, they help to investigate and to evaluate the effects of operational parameters on gasifier performance and product quality [5], [6].

Numerical and simulation studies of biomass gasification utilizing fluidized bed reactors have been the subject of many researchers in recent years [7]. Many numerical modelling and simulation studies have been published in the literature on the complicated behaviour of gas-solid flow, and fluidization hydrodynamics, to formulate the problems and develop solutions. Recently, computational fluid dynamics (CFD) has become a useful tool for understanding the hydrodynamics and transfer mechanisms in multiphase flow systems [8]. The primary advantage of the investigation of the fluidized bed through simulation is that it provides an accurate and affordable method for predicting hydrodynamic parameters compared to expensive experiments [9].

Moreover, CFD is a better option for experiments at high operating temperatures and pressures, which can be expensive but also can have security problems. CFD modelling to gas-solid fluidized beds has been divided into two approaches: Eulerian- Lagrangian approach and Eulerian-Eulerian approach. In the EulerianEulerian approach, both the gas and the solid are treated as a continuous phase. In contrast, in the EulerianLagrangian model, the gas is treated as a continuous phase and the solid as a discrete phase and the motion of each particle is tracked. In this study, the EulerianEulerian approach has been chosen for the CFD simulations. The two-fluid model (TFM) is widely known in the Eulerian-Eulerian approach treat mathematically the gas and solid phases as interpenetrating continua with similar conservation equations of mass and momentum. The coupling interfacial between gas and solid phases is realized by interphase forces, including the drag force, lift force, and virtual mass force. In bubbling fluidized beds, all forces other than the drag force can be neglected.

The drag force is an important term to describe the hydrodynamics of a fluidized bed in Eulerian-Eulerian approach, the importance of the drag force is due to its effect on the characteristics of the interaction between the gas and solid phases. two drag coefficient different correlations are available in the literature. For the drag force, different correlations are present in the literature. The models such as Syamlal O'Brien [10], Gidaspow [11] and Wen \& Yu [12] are frequently employed. These drag forces are based on the measurement of the 
terminal velocity of solid particles and the fraction of porosity in the fluidized bed.

Taghipour et al. [13] studied experimentally and computationally the hydrodynamics of a gas-solid fluidized bed using the Wen-Yu, Syamlal O'Brien, and Gidaspow drag models to determine the momentum exchange coefficients. The comparison of the different drag models with the experimental data indicates a good agreement in terms of pressure drops and bed expansion ratio.

Esmaili et al. [14] examined the effects of different drag models by utilizing a TFM model: Syamlal O'Brien, Gibilaro, Di Felice, Gidaspow, Wen-Yu, Arastoopour, Koch et al., and Zhang-Reese They found that all the models showed good qualitative agreement with the experimental data.

Moreover, Hamzehei et al. [15] investigated experimentally and computationally the particle size effect on heat transfer and hydrodynamics of a nonreactive gas-solid fluidized bed reactor using the TFM model and Syamlal O'Brien drag force. Pressure drops and temperature distribution predicted by the simulations at different particle sizes were in good agreement with experimental measurements at a superficial gas velocity higher than the minimum fluidization velocity. Simulation results also indicated that small bubbles were produced at the bottom of the bed. this study showed that the TFM model and the use of Syamlal O'Brien drag force can predict hydrodynamic and heat-transfer behaviour of gas-solid fluidized-bed reactors reasonably well. The drag model of Syamlal and O'Brien has been modified according to the minimum fluidization velocity of the particles. The modification procedure for the drag law is provided by Syamlal and O' Brien [16].

Zimmermann and Taghipour [17] performed a CFD simulation of the hydrodynamics and reaction kinetics of gas-solid fluidized beds containing fluid catalytic cracking (FCC) particles. The modification of the Syamlal-O'Brien drag law based on the minimum fluidization conditions, as a generalized method to decrease the drag, provides modelling predictions that are in reasonable agreement with the experimental data over the range in which the model was evaluated.

In the present work, CFD modelling has been carried out to study the hydrodynamic of bubbling fluidized bed reactor filled with spherical solid particles $275 \mu \mathrm{m}$ of diameter. The CFD simulation is based on the TFM model of Eulerian-Eulerian approaches where the solid phase properties are obtained by the kinetic theory of granular flow (KTGF). Momentum exchange coefficients were calculated using the modified Syamlal-O'Brien drag force. Simulation results were compared with the experimental data of Taghipour et al. [13] for model validation. Ultimately, the objective of the present paper is to develop the CFD parameters needed to accurately capture the hydrodynamic behaviour of a bubbling fluidized bed reactor, which will be valuable in designing commercial-scale fluidized bed reactors.

\section{Mathematical modelling}

The two-fluid model (TFM) based on the EulerianEulerian approach in combination with Kinetic Theory of Granular Flow (KTGF) is applied to simulate the hydrodynamics behaviour of gas-solid flow in a fluidized bed. In this model, the gas and solid phases were modelled as interpenetrating continua with similar conservation equations. The interactions between the two phases were expressed by the drag force added to the conservation equations. The gas was chosen for a primary phase and solid particles were selected for the second phase. The KTGF is used to define the proprieties of the solid phase through constitutive equations.

\subsection{Governing equations}

The governing equations of the problem include the conservation of mass, of momentum, and the solid phase fluctuating energy. Regarding an isothermal flow without chemical reactions between the two phases, the governing equation can be presented below:

Mass conservation equations of gas (g) and solid ( $\mathrm{s}$ ) phases:

$$
\begin{aligned}
& \frac{\partial}{\partial t}\left(\alpha_{g} \rho_{g}\right)+\nabla \cdot\left(\alpha_{g} \rho_{g} \vec{u}_{g}\right)=0 \\
& \frac{\partial}{\partial t}\left(\alpha_{s} \rho_{s}\right)+\nabla \cdot\left(\alpha_{s} \rho_{s} \vec{u}_{s}\right)=0
\end{aligned}
$$

The phases are interpenetrating with each other, so the volume of each phase could not be occupied by another phase, this means the sum of their volume fractions equal to unity.

$$
\alpha_{g}+\alpha_{p}=1
$$

Momentum equations of gas ( $\mathrm{g}$ ) and solid (s) phases:

$$
\begin{aligned}
& \frac{\partial}{\partial t}\left(\alpha_{g} \rho_{g} \vec{u}_{g}\right)+\nabla \cdot\left(\alpha_{g} \rho_{g} \vec{u}_{g} \vec{u}_{g}\right) \\
& =-\alpha_{g} \nabla P_{g}+\nabla \cdot \overline{\bar{\tau}}_{g} \\
& \quad+\alpha_{g} \rho_{g} \vec{g}+K_{g s}\left(\vec{u}_{s}-\vec{u}_{g}\right) \\
& \frac{\partial}{\partial t}\left(\alpha_{s} \rho_{s} \vec{u}_{s}\right)+\nabla\left(\alpha_{s} \rho_{s} \vec{u}_{s} \vec{u}_{s}\right) \\
& =-\alpha_{s} \nabla P_{g}-\nabla P_{s}+\nabla \cdot \overline{\bar{\tau}}_{s} \\
& +\alpha_{s} \rho_{s} \vec{g}+K_{s g}\left(\vec{u}_{s}-\vec{u}_{g}\right) \\
& K_{g s}=-K_{s g}: \text { interphase drag force coefficient }
\end{aligned}
$$

The solid phase fluctuating energy:

$$
\begin{aligned}
\frac{3}{2}\left[\frac{\partial}{\partial t}\left(\alpha_{s} \rho_{s} \Theta_{s}\right)+\right. & \left.\nabla\left(\alpha_{s} \rho_{s} \vec{u}_{s} \Theta_{s}\right)\right] \\
& =\left(-P_{s} \overline{\bar{I}}+\nabla \cdot \overline{\bar{\tau}}_{s}\right): \nabla \vec{u}_{s} \\
& +\nabla \cdot\left(\kappa_{\Theta_{s}} \nabla \Theta_{s}\right)+\varphi_{\Theta_{s}}-\gamma_{\Theta_{s}}
\end{aligned}
$$

\subsection{Constitutive equations}

Various closure models have been proposed to provide appropriate constitutive equations for 
multiphase flows based on the kinetic theory of granular flow. $e_{S S}$ is the restitution coefficient, which quantifies the elasticity of particle-particle collision resulting in kinetic energy dissipation. The restitution coefficient ranges from 0 for inelastic collisions with complete energy dissipation to 1 for fully elastic collisions with no energy dissipation [8]. The constitutive equations are given by:

Gas-phase stress tensor

$\overline{\bar{\tau}}_{g}=\alpha_{g} \mu_{g}\left[\nabla \vec{u}_{g}+\left(\nabla \vec{u}_{g}\right)^{T}-\frac{2}{3}\left(\nabla \cdot \vec{u}_{g}\right) \overline{\bar{I}}\right]$

Solid phase stress tensor

$\overline{\bar{\tau}}_{g}=\alpha_{s} \mu_{s}\left[\nabla \vec{u}_{s}+\left(\nabla \vec{u}_{s}\right)^{T}+\left(\frac{2}{3}-\frac{\lambda_{s}}{\mu_{s}}\right)\left(\nabla \cdot \vec{u}_{s}\right) \overline{\bar{I}}\right]$

Bulk viscosity

$\lambda_{s}=\frac{4}{3} \alpha_{s} \rho_{s} d_{s} g_{0}\left(1+e_{s s}\right)\left(\frac{\Theta_{s}}{\pi}\right)^{1 / 2}$

Solid shear viscosity

$\mu_{p}=\mu_{p}^{\text {coll }}+\mu_{p}^{k i n}+\mu_{p}^{f r}$

Solid collision viscosity [18]

$\mu_{s}^{\text {coll }}=\frac{4}{5} \alpha_{s} \rho_{s} d_{s} g_{0}\left(1+e_{S S}\right)\left(\frac{\Theta_{S}}{\pi}\right)^{1 / 2}$

Solid kinetic viscosity [19]

$\mu_{s}^{k i n}=\frac{10 \rho_{p} d_{p} \sqrt{\Theta_{s} \pi}}{96\left(3-e_{s s}\right) \alpha_{s} g_{0}}\left[1+\frac{4}{5}\left(1+e_{s s}\right) \alpha_{s} g_{0}\right]^{2}$

Solid frictional viscosity [20]

$$
\begin{aligned}
& \mu_{s}^{f r}=\frac{P_{s} \sin \emptyset}{2 \sqrt{I_{2 D}}} \\
& \begin{aligned}
I_{2 D}=\frac{1}{6}\left[\left(D_{s, 11}-D_{s, 22}\right)^{2}+\left(D_{s, 22}-D_{s, 33}\right)^{2}\right. \\
\\
\left.+\left(D_{s, 33}-D_{s, 11}\right)^{2}\right]+D_{s, 12}^{2} \\
+D_{s, 23}^{2}+D_{s, 31}^{2}
\end{aligned} \\
& D_{s, i j}=\frac{1}{2}\left(\frac{\partial \vec{u}_{s, i}}{\partial x_{j}}+\frac{\partial \vec{u}_{s, j}}{\partial x_{i}}\right)
\end{aligned}
$$

Solid phase pressure [19]

$P_{S}=\alpha_{s} \rho_{s} \Theta_{s}+2 \rho_{s}\left(1+e_{s S}\right) \alpha_{s} g_{0} \Theta_{S}$

Diffusion coefficient of granular temperature [11]

$$
\begin{array}{r}
k_{\Theta_{s}}=\frac{150 \rho_{s} d_{s} \sqrt{\pi \Theta_{s}}}{384\left(1+e_{s S}\right) g_{0}}\left[1+\frac{6}{5} \alpha_{s} g_{0}\left(1+e_{s s}\right)\right]^{2} \\
+2 \rho_{s} \alpha_{s}^{2} d_{s}\left(1+e_{s s}\right) g_{0} \sqrt{\frac{\Theta_{s}}{\pi}}
\end{array}
$$

Radial distribution function [20]

$g_{0}=\left[1-\left(\frac{\alpha_{s}}{\alpha_{s, \max }}\right)^{\frac{1}{3}}\right]^{-1}$

Collisional dissipation of energy [21]

$\gamma_{\Theta_{s}}=\frac{12\left(1+e_{s S}^{2}\right) g_{0}}{d_{s} \sqrt{\pi}} \rho_{s} \alpha_{s}^{2} \sqrt{\Theta_{s}^{3}}$

Transfer of kinetic energy [11]

$\phi_{g s}=-3 k_{g s} \Theta_{s}$

\subsection{Drag force:}

Syamlal O'Brien drag force:

$$
\begin{aligned}
& k_{g s}=\frac{3}{4} C_{D} \frac{\alpha_{g} \alpha_{s} \rho_{g}\left|\vec{u}_{s}-\vec{u}_{g}\right|}{d_{s} v_{r}^{2}}\left(\frac{R e_{s}}{v_{r}}\right)\left|\vec{u}_{s}-\vec{u}_{g}\right| \\
& C_{D}=\left(0.63+4.8\left(\frac{v_{r}}{R_{e_{s}}}\right)^{0.5}\right)^{2} \\
& v_{r, s} \\
& =0.5\left(A-0.06 R e_{s}\right) \\
& +\sqrt{\left(0.06 R e_{s}\right)^{2}+0.12 R e_{s}(2 B-A)+A^{2}} \\
& A=\alpha_{g}^{4.14}, \quad B=\left\{\begin{array}{cc}
P \alpha_{g}^{1.28} & \alpha_{g} \leq 0.85 \\
\alpha_{g}^{Q} & \alpha_{g}>0.85
\end{array}\right. \\
& P=0.8, \quad Q=2.65 \quad\left[\begin{array}{ll}
5
\end{array}\right.
\end{aligned}
$$

One of the significant terms to describe the hydrodynamics of the fluidized bed in a TFM model is the drag force. The importance of the drag force is due to its effect on the characteristics of the interaction between the two phases. The drag models used in this study to calculate the gas-solid momentum exchange coefficient are the modified Syamlal O'Brien. Syamlal and O'Brien [16] presented a modification of their original drag law to accurately simulate the minimum fluidization conditions. The modification of the Syamlal O'Brien drag law is based on the minimum fluidization conditions, commonly available experimental information for the specific parameters and materials [17]. The parameter $\mathrm{P}$ in Eq. (24) is related to the minimum fluidization velocity through the velocityvoidage correlation and the terminal Reynolds number by this objective function:

$$
u_{g}=R e_{t} \frac{\mu_{g} \alpha_{g}}{d_{s} \rho_{g}}=\left\{U_{m f}\right\}^{\text {experimental }}
$$


Where

$u_{g}=R e_{t} \frac{\mu_{g} \alpha_{g}}{d_{s} \rho_{g}}=U_{m f}^{\text {experimental }}$

$R e_{t}=v_{r, s} R e_{t, s}$

$R e_{t, s}=\left(\frac{\sqrt{4.8^{2}+2.52 \sqrt{\frac{4 A_{r}}{3}}}-4.8}{1.26}\right)^{2}$

$A_{r}=\frac{\left(\rho_{s}-\rho_{g}\right)}{\mu_{g}} d_{s}^{3} \rho_{g} \vec{g}$

$v_{r, s}=\frac{A+0.06 B R e_{t, s}}{1+0.06 R e_{t, s}}$

$Q=1.28+\frac{\log (P)}{\log (0.85)}$

Experimentally the minimum fluidization velocity is $0.065 \mathrm{~m} / \mathrm{s}$. The new parameters $\mathrm{P}$ and $\mathrm{Q}$ are taken respectably by 0.3 and 0.76 .

\section{Simulation methods}

\subsection{Boundary and initial conditions}

The geometry, mesh spacing and boundary conditions of the 2D fluidized bed reactor at the initial state are presented in Fig. 1. Like the experimental geometry of Taghipour et al.[13]. This reactor was 0.28 $\mathrm{m}$ in width and $1 \mathrm{~m}$ in height. Meshing used is a small square cell of $5 \times 5 \mathrm{~mm}$.

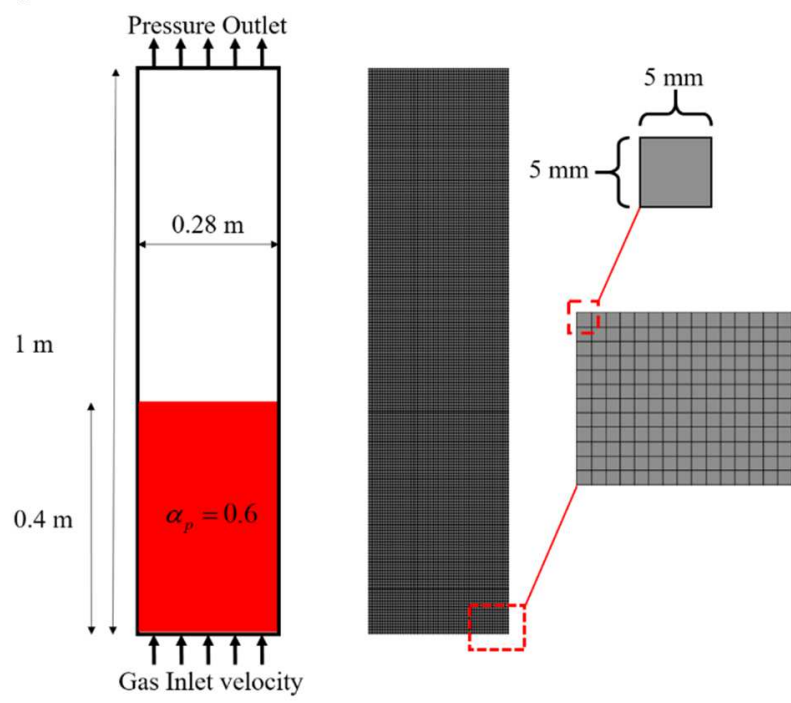

Fig. 1. Representative diagram of a $2 \mathrm{D}$ bubbling fluidised bed.

Initially, all velocities and volume fractions of each phase were specified. The particles solid were patched in the bed to the initial static bed height of $0.4 \mathrm{~m}$ with a volume fraction of 0.6. Air at ambient conditions was chosen for the gas phase and enter the bed from the bottom with the superficial gas velocity varying between $0.13-0.65 \mathrm{~m} / \mathrm{s}$. Spherical particles of sand were chosen for the solid phase, patched enter the bed with zero velocity. The product gas exits from the top of the bed where the pressure is set to atmospheric pressure.

At the wall, a no-slip boundary condition (the tangential normal velocities of the gases at the wall were set to zero) is applied for the gas and a Johnson and Jackson (1987) [22] slip boundary condition was applied for solid. At the outlet of the bed, the atmospheric pressure boundary condition was used.

\subsection{Simulation setup}

Two-dimensional (2D) simulations of the fluidized bed during quasi-steady-state conditions were performed. The TFM model described earlier was used for the analysis. The 2D computational domain was discretized using 11200 square cells. A time step of $0.001 \mathrm{~s}$ with 20 iterations per time step was also used. This number of iterations was found to be adequate to achieve convergence for the majority of time steps. the solution was run for $25 \mathrm{~s}$ to attain a quasi-steady-state condition, the time-averaging has been done between 10 $\mathrm{s}$ and $25 \mathrm{~s}$ to get the time-averaged value. Table 1 shows the values of all parameters that were used in the CFD simulations.

Table 1. CFD simulations parameters and proprieties.

\begin{tabular}{|l|c|}
\hline Description & Value \\
\hline Gas density & $1.225 \mathrm{~kg} / \mathrm{m}^{3}$ \\
\hline Gas viscosity & $1.8 \times 10^{-5} \mathrm{~kg} / \mathrm{m} . \mathrm{s}$ \\
\hline Superficial gas velocity & $0.13-0.65 \mathrm{~m} / \mathrm{s}$ \\
\hline Initial volume fraction & 0.6 \\
\hline Static bed height (Ho) & $0.4 \mathrm{~m}$ \\
\hline Specularity coefficient (Schaeffer) & 0.5 \\
\hline Packing limit (Syamlal and O'Brien) & 0.63 \\
\hline Friction parcking limit & 0.61 \\
\hline Angle of internal friction & $30^{\circ}$ \\
\hline Time steps & $0.001 \mathrm{~s}$ \\
\hline Convergence criteria & 0.0001 \\
\hline Maximum number of iterations & 20 \\
\hline Under relaxation factor & 0.6 \\
\hline Minimum fluidization velocity & $0.065 \mathrm{~m} / \mathrm{s}$ \\
\hline
\end{tabular}

The governing equations mentioned above are solved using the CFD commercial software ANSYS FLUENT 16.0. The discretized governing equations were solved by the finite volume method employing the Phase-Coupled SIMPLE (PC-SIMPLE) which is an extension of the SIMPLE algorithm of Patankar [23] for multiphase flow, is used for the treatment of pressurevelocity coupling. The steps of this algorithm for CFD simulation are shown in Fig. 2. For the unsteady 
formulation, first-order scheme is used for discretization of convective terms was used for all differential equations. the convergence criterion for maximum residual between two iterations was set to 0.0001 . Relaxation factors of 0.6 were adopted for all gas-solid flow quantities. All simulations were performed on a computer workstation of 12 processors i7-8750H CPU @ $2.20 \mathrm{GHz} 64$ bit and 8GB of RAM.

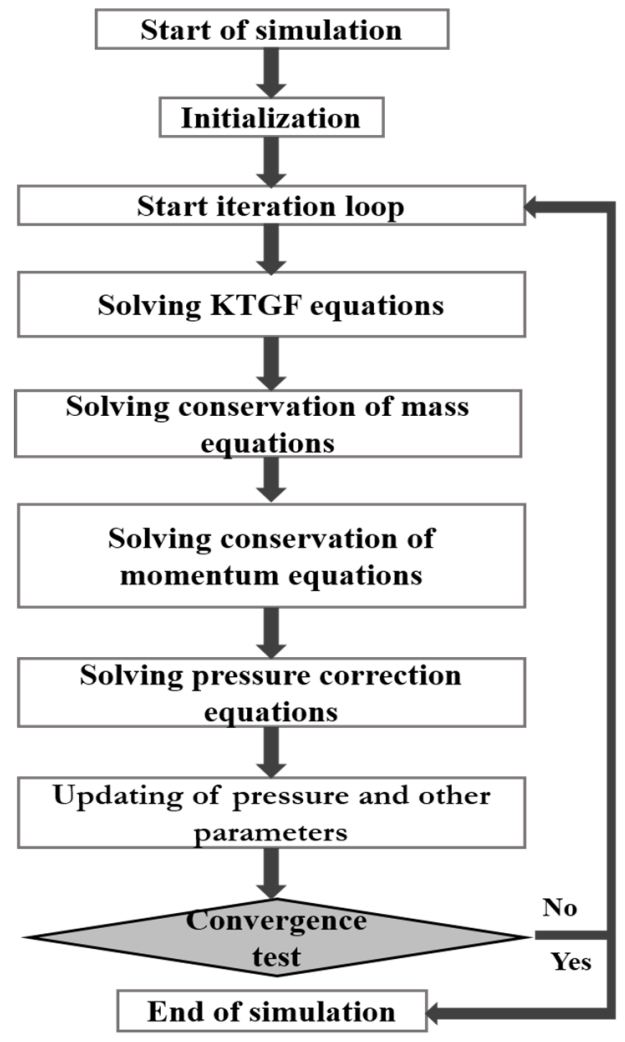

Fig. 2. CFD simulation algorithm.

\section{Result and discussion}

As discussed, the emphasis in this work is to understand the hydrodynamic behaviour of gas-solid flow in a bubbling fluidized bed reactor.

In the following section, the perform the numerical simulation, it was defined ten gas velocities $(0.065$, $0.13,0.26,0.38,0.46,0.52,0.6 \mathrm{~m} / \mathrm{s}$ ) and were compared all simulation results with the experimental data of Taghipour et al. [13].

\subsection{Pressure drops}

Fig. 3 shows the simulations predicted pressure drop by modified drag with different gas superficial velocities. The black continuous line represents experimental data realized by Taghipour et al. [13]. A linear increase in pressure drop is noted up to the minimum fluidization velocity of $0.065 \mathrm{~m} / \mathrm{s}$. Also, an increase in gas velocity leads to an increment in pressure drop up to a maximum value of $6200 \mathrm{~Pa}$ in velocity of $0.6 \mathrm{~m} / \mathrm{s}$, this velocity represents the terminal fluidization velocity. The figure illustrates that the pressure drops obtained by the CFD simulations is a little different from the experimental data, especially at a low gas surface velocity $u_{g}<0.38 \mathrm{~m} / \mathrm{s}$. The possible reason may be due to the type of air distributor used experimentally (is not a uniform gas inlet), the configuration of the air distributor in the experiment results in a higher pressure at the inlet, which causes a small drop in pressure at the inlet. As gas superficial velocity increases, the correspondence between the simulation results and the experimental data is more evident. In short, with increasing fluidization, the congruence between the simulation results and the experimental data also increases.

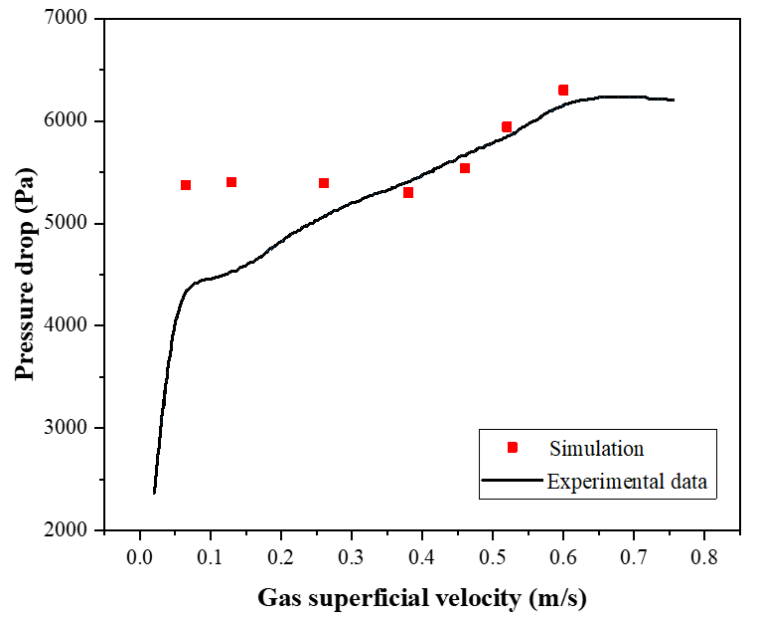

Fig. 3. Pressure drop at different gas superficial velocities.

\subsection{Bed expansion ratio}

Fig. 4 present the comparison of experimental results and CFD simulations predicted bed expansion ratio with different superficial gas velocities. in comparison with the experimental data, CFD simulation results are showing the correct trend of increased bed expansion at different gas superficial velocities with less than $2 \%$ error. The bed expansion ratio increases with the superficial gas velocity due to the increase of the forces of repulsion affecting the solid particles.

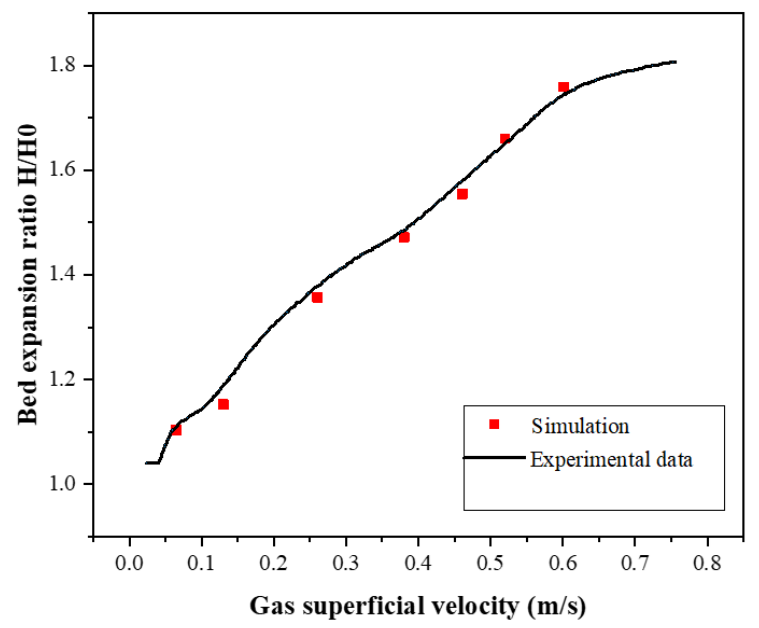

Fig. 4. Bed expansion ration at different gas superficial velocities 
A

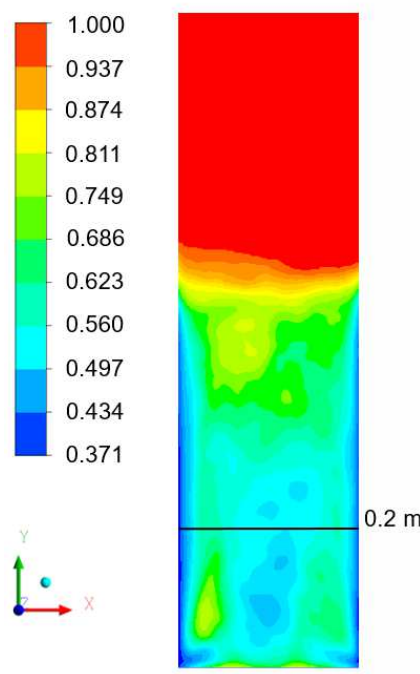

B

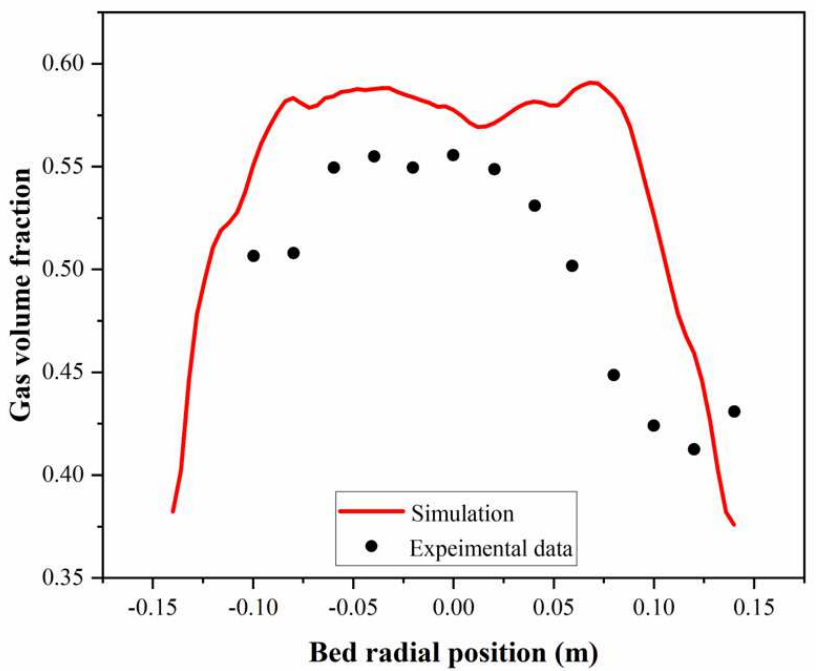

Fig. 5. Time average gas volume fraction: (A) profile of average gas volume fraction, (B) comparison of simulation and experimental data of average gas volume fraction.

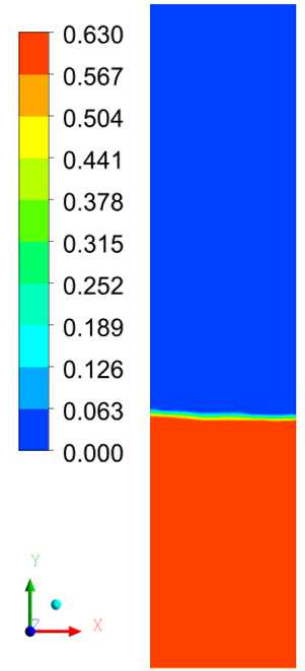

$0.065 \mathrm{~m} / \mathrm{s}$

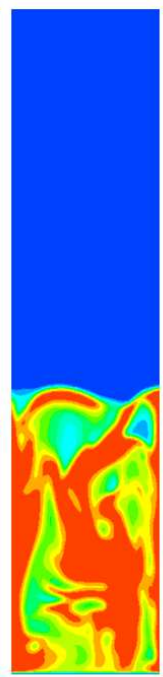

$0.13 \mathrm{~m} / \mathrm{s}$

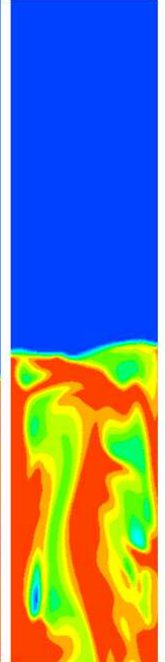

$0.26 \mathrm{~m} / \mathrm{s}$

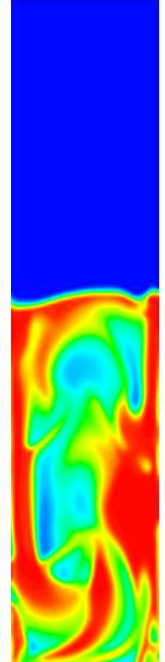

$0.38 \mathrm{~m} / \mathrm{s}$

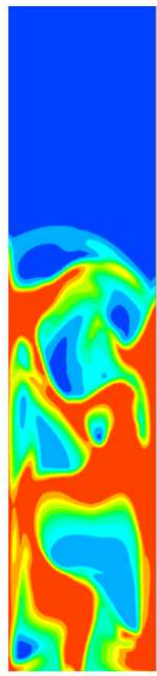

$0.46 \mathrm{~m} / \mathrm{s}$

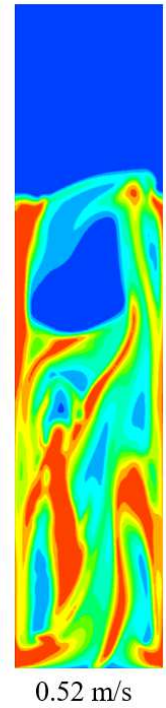

Fig. 6. The contour plots of solid volume fraction at 25 s for different gas superficial velocities.

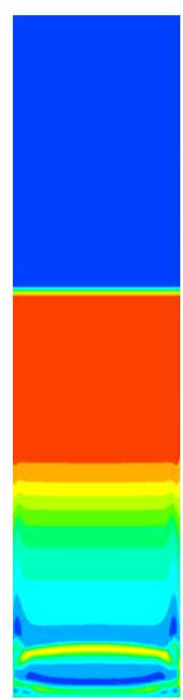

$0.5 \mathrm{~s}$

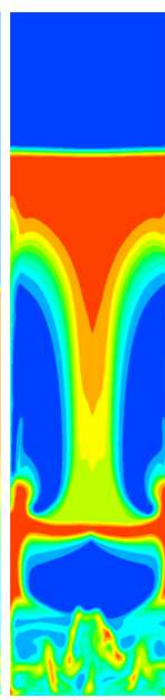

$1 \mathrm{~s}$

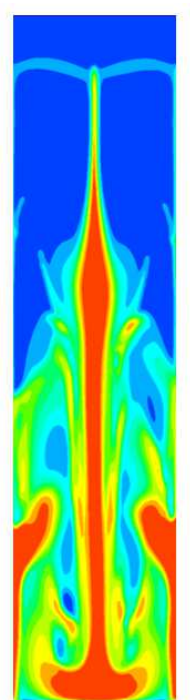

$1.5 \mathrm{~s}$

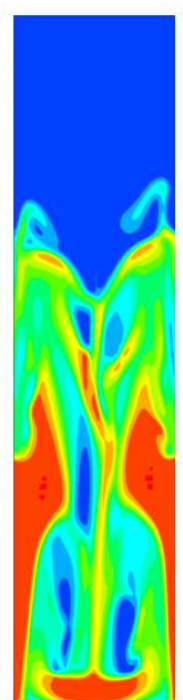

$1.8 \mathrm{~s}$

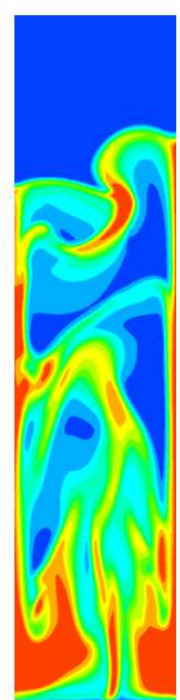

$2.5 \mathrm{~s}$

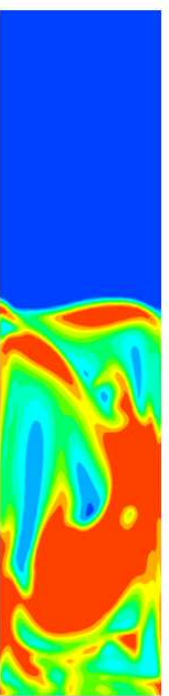

$10 \mathrm{~s}$

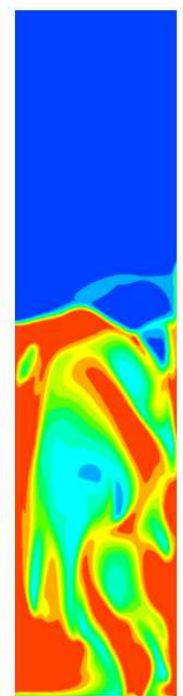

$15 \mathrm{~s}$

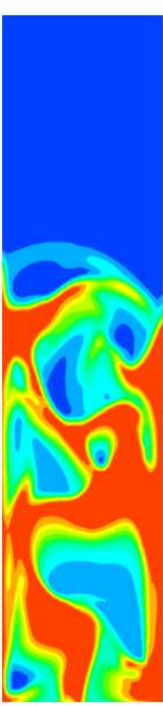

$25 \mathrm{~s}$

Fig. 7. CFD simulations predicted instantaneous solid volume fraction contours for the superficial gas velocity of $0.46 \mathrm{~m} / \mathrm{s}$. 


\subsection{Time average gas volume fraction profile}

Fig. 5A shows the gas volume fraction contours, and shows a low gas volume fraction near the walls of about 0.37 , in the central region of the fluidized bed, the simulation was able to capture a slight reduction in gas volume fraction due to the downward flow of the particles along the walls. The top bed section gives an almost uniform profile and appears less dynamic than the bottom section of the bed. The gas volume fraction contour results in an approximately symmetric flow profile. This flow structure composed of multiple turbulent cells causes the particle concentration to be uniform throughout the bed due to uniform mixing.

The simulation time-average gas volume fraction is compared to the experimental results in Fig. 5B for $u_{g}=$ $0.46 \mathrm{~m} / \mathrm{s}$ at $\mathrm{y}=0.2 \mathrm{~m}$. Simulation results show deviations from experimental results near the walls of the bed and greater conformity in the centre of the bed. Also, can observe that the minimum gas volume fraction values are near the walls of the bed for both the simulation results and the experimental data. This indicates that the solids concentration is higher near the walls of the bed. This is due to the de-fluidization phenomena of the solid particles occurring near the wall region, while fluidization occurs in the centre of the bed where the average gas volume fraction has moderate to maximum values of 0.58 . Consequently, a visible coreannulus structure is produced by CFD simulation. A similar observation was also made by the simulation results of Taghipour et al. [13], Rashid et al.[24] and Loha et al. [25].

\subsection{Contour of solid volume fraction}

Fig. 6 shows the solid volume fraction contour for the different gas velocities, i.e., at $0.065,0.13,0.26$, $0.38,0.46,0.52$ and $0.6 \mathrm{~m} / \mathrm{s}$ full and closure view at the bed area. the bed starts to expand at a small scale but almost the bed height is equal to the static bed height. For the gas velocity of $0.13 \mathrm{~m} / \mathrm{s}$, the bubbles appear only at the surface and are at the static bed height with little bed expansion. At the gas velocity of $0.38,0.46,0.52$ and $0.6 \mathrm{~m} / \mathrm{s}$ larger bubbles appear and increase of bed expansion. It means that the bubble flow regime has appeared.

The contour plots of the solid volume fractions corresponding to different time steps from the initial condition $\mathrm{t}=0 \mathrm{~s}$ and continuing for $\mathrm{t}=25 \mathrm{~s}$ of complete fluidization are illustrated in Fig. 7. Initially, the formation of bed height bubbles until it stabilizes at a stable bed height. it can be seen that the particles show an axisymmetric distribution. The bubbles started to form at the inlet of the air and expanded as they moved upward due to the surface tension gradient. All bubbles formed ultimately tend to migrate to the bed surfaces and to collapse. The movements of the bubbles, when colliding with the stationary particles, transfer kinetic energy and make them move in different directions depending on the height of the bed, thus establishing the fluidization phenomenon. In addition, it can be observed that the expansion of the bed remains quasi-continuous after the time 10 s can be seen the quasi-steady-state in $10 \mathrm{~s}$, therefore the fluidization in steady-state.

\section{Conclusion}

A TFM model based on the Eulerian-Eulerian approach integrating the kinetic theory of granular using ANSYS Fluent 16.0 CFD software was capable of predicting the hydrodynamics of gas-solid behaviour in a fluidized bed reactor. Comparison of the model predictions, using the modified Syamlal O'Brien drag force and experimental data, the CFD simulation indicated reasonable agreement on the bed expansion ratio. The pressure drops predictions by the CFD situation deviate at low superficial gas velocities. in addition, for the higher superficial velocity, the congruence between the simulation results and the experimental data is more increases. On balance, as a necessary effort, this work used the numerical modelling method as an effective tool to develop the vital hydrodynamic correlation instead of using the state-ofthe-art experimental measurements, which holds the promise of saving sensing expenses and assisting in the design and scaling of processing devices.

\section{Nomenclature}

\section{Notation}

$\begin{array}{llc}C_{D} & \text { Drag coefficient } & \\ d & \text { diameter } & \mathrm{m} \\ e_{S S} & \text { Restitution coefficient } & \\ g & \text { Acceleration due to gravity } & \mathrm{m}^{2} / \mathrm{s} \\ g_{0} & \text { Radial distribution coefficient } & \\ H & \text { Expanded bed height } & \mathrm{m} \\ H_{0} & \text { Static bed height } & \mathrm{m} \\ \overline{\bar{I}} & \text { Identity Tensor } & \\ I_{2 D} & \text { Second invariant of the deviatoric } & \\ & \text { stress tensor } & \\ k_{\theta_{s}} & \text { Diffusion coefficient for granular } & \mathrm{kg} / \mathrm{s} \mathrm{m} \\ & \text { energy } & \\ k_{g s} & \text { Interphase momentum transfer } & \mathrm{N} \mathrm{s} \mathrm{m} \\ & \text { coefficient } & \\ P_{k} & \text { Pressure } & \mathrm{Pa} \\ R e_{s} & \text { Particle Reynolds number } & \\ t & \text { Time } & \mathrm{s} / \mathrm{s} \\ \vec{u}_{k} & \text { Velocity } & \end{array}$

\section{Greek letters}

$\begin{array}{llc}\rho_{k} & \text { Density } & \mathrm{kg} / \mathrm{m}^{3} \\ \alpha_{k} & \text { Volume fraction } & \\ \mu_{k} & \text { viscosity } & \mathrm{Pas}\end{array}$


$\Theta_{s} \quad$ Granular temperature $\quad m^{2} s^{-2}$

\section{Subscripts}

$\begin{array}{ll}g & \text { Gas index } \\ m f & \text { Minimum fluidization } \\ s & \text { Solid index }\end{array}$

\section{References}

1. R. Khezri, W. Azlina, and H. T. -, Int. J. Biomass 5, 6 (2016).

2. F. Aiche, S. Belaadi, A. Lalaoua, A. S. Berrouk, and A. Azzi, Proc. Inst. Mech. Eng. Part E J. Process Mech. Eng. 235, 1005 (2021).

3. S. Sansaniwal, M. Rosen, and S. Tyagi, Renew. Sustain. Energy 80, 23 (2017).

4. R. Khezri, W. W. A. K. Ghani, and S. Masoudi Soltani, Processes 7, 524 (2019).

5. Y. Liu, P. Huo, X. Li, and H. Qi, Fuel Process. Technol. 203, 106363 (2020).

6. D. Kunii and O. Levenspiel, Fluidization Engineering (1991).

7. N. Couto, V. Silva, E. Monteiro, P. Brito, and R. Rouboa, Procedia Energy 36, 591 (2013).

8. P. Li, X. Lan, C. Xu, G. Wang, C. Lu, and J. Gao, Particuology 7, 269 (2009).

9. T. A. Bin Rashid, L. T. Zhu, and Z. H. Luo, Adv. Powder Technol. 31, 2723 (2020).

10. M. Syamlal and T. J. O'Brien, AIChE Symp 85, 22 (1989).

11. D. Gidaspow, R. Bezburuah, and J. Ding, 7th Fluid. Conf. 75 (1992).

12. C. Y. Wen, Chem. Eng. Prog. 62, 100 (1996).

13. F. Taghipour, N. Ellis, and C. Wong, Chem. Eng. Sci. 60, 6857 (2005).

14. E. Esmaili and N. Mahinpey, Adv. Eng. Softw. 42, 375 (2011).

15. M. Hamzehei and H. Rahimzadeh, Ind. Eng. Chem. Res. 48, 3177 (2009).

16. M. Syamlal and T. . O'Brien, U.S. Dep. Energy, Off. Foss. Energy, Natl. Energy Technol. Lab. Morgantown, WV (1987).

17. S. Zimmermann and F. Taghipour, Ind. Eng. Chem. Res. 44, 9818 (2005).

18. M. Syamlal, W. Rogers, and T. J. O`Brien, MFIX Documentation Theory Guide (1993).

19. D. GIDASPOW, Multiphase Flow and Fluidization (Academic Press, 1994).

20. S. Ogawa, A. Umemura, and N. Oshima, Zeitschrift Für Angew. Math. Und Phys. ZAMP 31, 483 (1980).

21. C. Lun, S. Savage, ... D. J.-J. of fluid, and U. 1984, J. Fluid Mech. 140, 223 (1984).
22. P. C. Johnson and R. Jackson, J. Fluid Mech. 176, 67 (1987).

23. S. V. PATANKAR and D. B. SPALDING, $A$ Calculation Procedure for Heat, Mass and Momentum Transfer in Three-Dimensional Parabolic Flows (Pergamon Press, Ltd, 1983).

24. T. A. Bin Rashid, L. T. Zhu, and Z. H. Luo, Adv. Powder Technol. 31, 2723 (2020).

25. C. Loha, H. Chattopadhyay, and P. K. Chatterjee, Chem. Eng. Sci. 75, 400 (2012). 